\section{Aortic root in competitive sports: eyes on the older athlete}

\author{
Harald T Jorstad, ${ }^{\ominus}$ Maarten Groenink
}

Individuals with a suspicion of aortic disease are discouraged to take part in most competitive sports. ${ }^{1}$ In the more prevalent and syndromic forms, such as Marfan syndrome (prevalence 1: 5000), a sports examination with echocardiography may reveal this condition, with great consequences for the fate (and especially career) of a young athlete. From the point of view of the most recent guidelines, ${ }^{12}$ the prevention of aortic dissection is emphasised as the main task of clinicians. Cut-off values and $\mathrm{z}$-scores are supplied to identify those that could be at risk for a pathophysiological process leading to cardiovascular morbidity and mortality. At the same time, sports physicians are often struggling with the cases that may be less clear, where slight aortic dilatation may be considered physiological. While the initial steps are evidentthoroughly investigate if there are any signs of underlying diseases such as Marfan syndrome or related conditionsin individuals with a mild dilatation of the aortic root without any other signs of an underlying disease, the next steps to be taken can be less clear. The increased availability of genetic panels to detect inborn predispositions for a wide range of aortic disorders may tempt sports physicians to reach for this diagnostic modality. However, uncertain pathogenicity and extreme variable penetrance of found mutations may just further obscure the situation.

Current data from different groups of athletes suggest that training on its own has only a limited impact on physiological aortic root remodelling (figure 1). ${ }^{34}$ This impression is strongly reinforced by the results presented by Gati et $a l^{5}$ in this issue of Heart, who echocardiographically investigated aortic root diameters in 3781 athletes aged $19 \pm 5.9$ years, in whom hereditary thoracic aortic disease was definitely ruled out. These aortic dimensions were similar to a control population of 806 individuals when

Departments of Cardiology and Radiology, Amsterdam University Medical Centre, location AMC, Amsterdam, $\mathrm{NH}$, The Netherlands

Correspondence to Dr Maarten Groenink, Cardiology, Academic Medical Center Amsterdam, Amsterdam 1105AZ, The Netherlands; m.groenink@ amc.uva.nl corrected for BSA. The 99th percentile in the athlete population was $40 \mathrm{~mm}$ in men and $38 \mathrm{~mm}$ in women, and only five men $(0.17 \%)$ and six women $(0.4 \%)$ had an aortic root diameter above the 99th percentile. After a mean follow-up of $5 \pm 1.5$ years, none of these athletes showed a significant increase in their aortic root dimensions, and there were no aortic events in the entire athlete population. In short, a severely dilated aortic root without an underlying disease is seldom found in young athletes, and in athletes with mild enlargement, there seems to be no clinically important progression of the dilatation, despite of ongoing participation in sports over a period of approximately 5 years. The authors therefore suggest that 'there is scope for being more liberal in athletes with a slightly enlarged aortic diameter in the future although annuals assessments are recommended'.

A number of important factors should be kept in mind when assessing and performing follow-up of the athlete's aortic root. First, when measuring the aortic root using two-dimensional echocardiography in the parasternal long axis, the assumption is made that this is the real representation of the sinus of valsalva. However, a cross-sectional echo plane could well inadvertently intersect the vessel obliquely or not intersect the midline of the vessel, leading to an

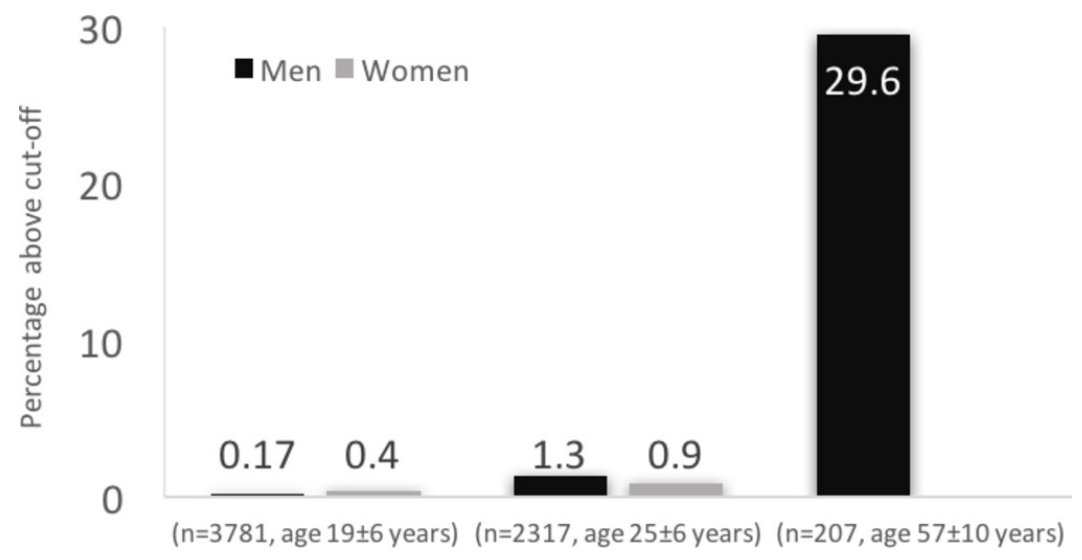

Figure 1 Prevalence of aortic dilatation in athletes in three age categories. Legend: prevalence of aortic dilatation above predefined cut-offs according to study. Gati et al $\beta^{3}(n=3781$, age $19 \pm 6$ years): aortic root 99th percentile: men: $40 \mathrm{~mm}$, women: $38 \mathrm{~mm}$. Pelliccia et al ( $n=2317$, age $25 \pm 6$ years): aortic root 99th percentile: men: $40 \mathrm{~mm}$, women: $34 \mathrm{~mm}$. Gentry et al $(n=207$, age $57 \pm 10$ : years): ascending aorta $40 \mathrm{~mm}$ in men. underestimation or overestimation of the diameter. ${ }^{6}$ Considering that $2 \mathrm{~mm}$ or even $1 \mathrm{~mm}$ growth in aortic root diameter should be viewed as highly suspicious in athletes, as suggested by Gati et al, reproducibility of findings is of importance when performing follow-up.

Second, the epidemiology of aortic dilatation teaches us that most dilatation takes place later in life. Gentry et al have previously shown that the prevalence of dilatation of the ascending aorta as measured by computer tomography among 207 middleaged ( $57 \pm 10$ years), male, former American-style football players was almost 30\% using $>40 \mathrm{~mm}$ as a cut-off and $9 \%$ using $\geq 45 \mathrm{~mm}$ as a cut-off (figure 1). ${ }^{7}$ This is in sharp contrast to the numbers reported in young athletes. Is this primarily driven by the changes in lifestyle and progression of classical cardiovascular risk factors such as hypertension after cessation of a professional sports career, or are these changes secondary to sports-related factors, such as repetitive injury to an aorta with a propensity to dilate or to the demands of high-dynamic sports performance? The medium-term follow-up performed in the study by Gati et al serves to reassure us for the early parts of an athlete's career, but studies are urgently needed to investigate and cessation of a professional sports career, the associated changes in lifestyle and ageing.

Third, while sudden cardiac death due to aortic diseases is rare in the elite athlete, ${ }^{8}$ the question remains if the enlarged aorta of an athlete carries the same propensity for dissection as in the general population, both in youth and later in life. While a liberal approach is the complex interplay between detraining 
probably warranted in the young athlete with mild aortic root dilatation, clinicians should remain vigilant in athletes at the end of their sports career and in later life. Prospective, longitudinal data are needed across a wide variety of sports and ages for further understanding the interaction between health and sports and to identify those individuals in whom too much of a good thing, in this case sports, might actually be a bad thing.

Correction notice Since this paper was first published online, the author name Harald, HT Jorstad has been updated to Harald T Jorstad. This article has been corrected since it first published online. The open access licence type has been amended.

Contributors Both authors contributed equally.

Funding The authors have not declared a specific grant for this research from any funding agency in the public, commercial or not-for-profit sectors.

Competing interests None declared.

Patient consent for publication Not required.

Provenance and peer review Commissioned; internally peer reviewed.

Data sharing statement No additional data are available.
(6)

\section{OPEN ACCESS}

Open access This is an open access article distributed in accordance with the Creative Commons Attribution 4.0 Unported (CC BY 4.0) license, which permits others to copy, redistribute, remix, transform and build upon this work for any purpose, provided the original work is properly cited, a link to the licence is given, and indication of whether changes were made. See: https:// creativecommons.org/licenses/by/4.0/.

(c) Author(s) (or their employer(s)) 2019. Re-use permitted under CC BY. Published by BMJ.

\section{(D) Check for updates}

To cite Jorstad HT, Groenink M. Heart 2019;105:900-901.

\section{Sinked}

- http://dx.doi.org/10.1136/heartjnl-2018-314288

Heart 2019:105:900-901.

doi:10.1136/heartjnl-2019-314759

\section{REFERENCES}

1 Braverman AC, Harris KM, Kovacs RJ, et al. Eligibility and Disqualification Recommendations for Competitive Athletes With Cardiovascular Abnormalities: Task Force 7: Aortic Diseases, Including Marfan Syndrome: A Scientific Statement From the American Heart Association and American College of Cardiology. Circulation 2015;132:e303-9.

2 Erbel R, Aboyans V, Boileau C, et al. 2014 ESC Guidelines on the diagnosis and treatment of aortic diseases: Document covering acute and chronic aortic diseases of the thoracic and abdominal aorta of the adult. The Task Force for the Diagnosis and Treatment of Aortic Diseases of the European Society of Cardiology (ESC). Eur Heart J 2014;35:2873-926.

3 Pelliccia A, Di Paolo FM, De Blasiis E, et al. Prevalence and clinical significance of aortic root dilation in highly trained competitive athletes. Circulation 2010;122:698-706.

4 Iskandar A, Thompson PD. A meta-analysis of aortic root size in elite athletes. Circulation 2013;127:791-8.

5 Gati S, Malhotra A, Sedgwick C, et al. Prevalence and progression of aortic root dilatation in highly trained young athletes. Heart 2019;105:920-5.

6 Vonder M, Willems TP, van Melle JP, et al. True diameter of vessels. J Am Soc Echocardiogr 2014;27:448-9.

7 Gentry JL, Carruthers D, Joshi PH, et al. Ascending Aortic Dimensions in Former National Football League Athletes. Circ Cardiovasc Imaging 2017;10.

8 Maron BJ, Doerer JJ, Haas TS, et al. Sudden deaths in young competitive athletes: analysis of 1866 deaths in the United States, 1980-2006. Circulation 2009;119:1085-92. 